\title{
Binding Partners of 14-3-3 (YWHA) Protein Isoforms among Mammalian Species, Tissues, and Developmental Stages
}

\author{
Taylor R. Covington, Santanu De*
}

Department of Biological Sciences, Halmos College of Arts and Sciences, Nova Southeastern University, 3301 College Avenue, Fort Lauderdale, Florida 33314, U.S.A

* Corresponding Author email: sde@nova.edu

Article History

Received: 26 December 2020

Accepted: 15 March 2021

Published: 27 March 2021

Student(s)

- $\quad$ Taylor R. Covington

Academic Year: 2020-2021/Fall

Course Level: Bachelor

Course Name: B.S. (Biology)

Course year: Final Year

Mentar(s)

- Dr. Santanu De

\section{ABSTRACT}

The 14-3-3 (YWHA or Tyrosine 3-Monooxygenase/Tryptophan 5-Monooxygenase Activation proteins) are a family of abundant, highly conserved, ubiquitous, acidic, and homologous proteins expressed in most eukaryotes ranging from plants to animals, including humans, important in regulating a multitude of cellular processes such as signal transduction, cell cycle, protein trafficking, metabolism, apoptosis, and development. Mammals have been noted contain seven isoforms of these proteins (beta, epsilon, eta, gamma, sigma, tau/theta, and zeta), encoded by separate genes. The 14-3-3 proteins are known to interact with over 200 binding partners in isoform-specific, tissue-specific, and developmental stage-specific ways. The present review article encapsulates previously published research articles that report 14-3-3interactors, and investigates isoform-specific interactions within a wide array of mammalian species, cells, tissues, organs, and developmental stages. Of the hundreds of binding partners of 143-3 discovered till date, this paper focuses on analyzing selected, representative interactors with key functional roles. The study would help a better understanding of isoform-specific interactions of this critical protein family in mammals.

Keywords: 14-3-3 protein, YWHA, 14-3-3 isoforms, binding partners, mammals

\section{Introduction}

The interactions between a protein and its binding partners are essential in regulating a plethora of cellular events. An important family of such regulatory proteins in eukaryotes is the widely studied 14-3-3 or YWHA (Tyrosine 3-Monooxygenase/Tryptophan 5-Monooxygenase Activation protein). In mammals, there are seven 14-3-3 isoforms that are encoded by separate genes and named according to Greek alphabets: beta $(\beta)$, epsilon $(\varepsilon)$, eta $(\eta)$, gamma $(\gamma)$, sigma $(\sigma)$, tau/theta $(\tau / \theta)$, and zeta $(\zeta)$. These proteins have been shown to interact with over 700 other proteins [1], allowing them to regulate diverse physiological processes such as the cell cycle, cell differentiation, and apoptosis [2]. These binding interactions involving the 14-3-3 proteins occur across all mammalian species, differing on the basis of cell types, tissues, organs, and developmental stages. 
The 14-3-3 proteins can exist as homodimers, heterodimers, or monomers to participate in both phosphorylation-dependent and -independent binding depending on the target protein. A primary interaction occurs when the main chain and phosphate group of the target protein binds to the conserved binding site on the 14-3-3 protein, and the secondary interaction then occurs when the remainder of the target protein binds to the rest of the 14-3-3 protein through various rearrangements that aid in contact formation. These proteins have isoform-specific binding partners with varying size, shape, and sequence due to the conformational flexibility in the binding site and in dimerization [3]. This paper examines previously published research on the 14-3-3 isoforms, compiling and characterizing representative examples of notable 14-3-3-binding partners in mammals by species, locations, and developmental stages. The purpose is to create a summative resource that provides this information from other studies in a more concise format focused on binding partners.

\section{Binding Partners of 14-3-3 in Various Mammalian Species}

The seven 14-3-3 isoforms that are found in mammals have variable levels of expression, functions, and protein interactions in diverse species. The most abundant research on 14-3-3-interacting proteins is available on humans (Homo sapiens), mice (Mus musculus), and cows (Bos taurus). However, one or more 143-3 isoforms along with their binding partners have also been detected in a wide variety of other mammalian and non-mammalian species [4-10].

\subsection{Humans}

The 14-3-3 proteins have been shown to interact with many different proteins in humans and effect a multitude of cellular mechanisms. O-linked $\beta$-N-acetylglucosamine (O-GlcNAc) is a modification made to proteins in the nucleus, cytoplasm, and mitochondria that impacts what biological processes they participate in. Research by Toleman et al. demonstrates that 14-3-3 isoforms bind to O-GlcNAc modified regions in human cells to regulate those biological processes by integrating the modifications with other signaling pathways within the cell [11]. More than 200 phosphoproteins were obtained by 14-3-3-affinity purification, indicating roles in governing cellular trafficking, proliferation, and metabolism [12].

More isoform-specific interactions that have also been studied in human cells. The 14-3-3 beta isoform binds to $\beta 1$ - integrin, a cell surface protein that links the extracellular matrix to the actin cytoskeleton, and overexpression of this interaction can stimulate cellular migration in non-neuronal cells. Its interaction with the Raf-1 proto-oncogene can also play a role in promoting cellular migration [13]. This report showed that 14-3-3 epsilon binds to Doublecortin (Dcx), a microtubule binding protein, in a phosphorylation-specific manner. Overexpression of this interaction may disrupt organization of microtubules in the cell, but when Dcx is stabilized with 14-3-3 epsilon, it results in normal microtubule formation. On the other hand, this article noted that 14-3-3 tau inhibits Rho GDP-disassociation inhibitor $\alpha$ (RhoGDI $\alpha$ ), a protein that prevents GDP/GTP cycling. This interaction promotes activation of cell division control 42 (Cdc42) which then converts signals to downstream interactions and initiates other cellular effects.

\subsection{Mice}

A lot of the research on 14-3-3 proteins and their binding partners have been performed on mice. All seven isoforms are expressed in oocytes as well as eggs of mice; however, while all of them seem to interact with M-phase inducer phosphatase 2 (CDC25B) in mouse oocytes and eggs, 14-3-3 eta and 14-3-3 epsilon are not necessary for oocyte maturation, fertilization, and early embryonic development [14-24]. The 14-3-3 eta isoform appears to interact with $\alpha$-tubulin at the metaphase II spindles of mouse eggs [14, 15, 25-27]. In the granulosa cells of mice, 14-3-3 tau binds to follicle stimulating hormone (FSH) receptors to impact cell signaling [28]. In this study, it was also found that 14-3-3 theta and zeta interact with cyclin A1 (Ccna1), inhibiting cell death. A binding partner of the 14-3-3 sigma protein is the keratin 14 (K14) protein that was identified in the epidermis of mouse skin [29]. Intracellular regulation of peptidylarginine deiminase type VI (PADI6) may be regulated by its phosphorylation and/or binding with 14-3-3 [30]. 
Taylor R. Covington and Santanu De, Adv. J. Grad. Res.; Vol. 10, Issue 1, pp: 16-22, July 2021

\subsection{Cows}

The family of 14-3-3 proteins was first discovered in bovine brain samples [31]. This research has since been extended to a wide variety of organisms, organs, tissues, and cells. Tyrosine hydroxylase (TH) from the midbrain was found to be regulated by the 14-3-3 proteins [32]. All seven mammalian isoforms bind to phosphorylated TH as homodimers, but the 14-3-3 gamma homodimer was determined to be the strongest binding partner. Another study was done on bovine mammary epithelial cells, and the initiation factor eIF5 was identified as a binding partner of 14-3-3 gamma. This interaction was shown to regulate the translation of $\beta$-casein during the synthesis of milk proteins [33].

\section{Binding Partners of 14-3-3 in Various Cells, Tissues, and Organs}

The seven 14-3-3 isoforms that are found in mammals have variable levels of expression, functions, and protein interactions in numerous cells, tissues, and organs. Binding partners within cancer cells will be addressed, as well as binding partners within muscle tissue, reproductive tissues, the brain, and skin.

\subsection{Cancer Cells}

The 14-3-3 proteins often have pro-apoptotic effects within cancerous cells. The 14-3-3 sigma isoform binds to cell division cycle 2 (CDC2) complexes, genes that encode for cyclin-dependent kinase 1 (CDK1), and sequesters them in the cytoplasm to prevent cell cycle progression so that the cell has time to repair damage to its DNA [13]. Without this binding interaction, damaged DNA or mutations may be able to progress through replication and cause cancer. In a study on neuroblastoma cells, it was noted that the interaction between 14-3-3 and BRCA 1-associated protein 1 (BAP1) is responsible for a delay in the $\mathrm{S}$ and $\mathrm{G} 2 / \mathrm{M}$ phases of the cell cycle and the release of $\mathrm{Bcl}-2$-associated $\mathrm{X}$ protein (BAX), an apoptotic inducing protein [34].

On the other hand, 14-3-3 interactions can also aid in the survival of cancer cells. Checkpoint kinase 1 (Chk1) is a binding partner of 14-3-3 in which the interaction prevents the ability of E2 transcription factor (E2F) proteins to repress transcription in atypical cells and thus, leads to the continuation of cancer cell growth [35]. Research on anaplastic thyroid carcinoma cells by Zhong et al. (2020) shows that 14-3-3 epsilon and gamma are binding partners of the lymphocyte adaptor protein (LNK) [36]. Overexpression of LNK and this interaction increases its anti-apoptotic functions, enabling the cancer cells to survive.

\subsection{Muscle Tissue}

The human small heat shock protein (HSPB6) has the capacity to bind with 14-3-3, and this interaction is involved in triggering smooth muscle relaxation [37]. Through this work, it was determined that binding occurs between dimers of all seven 14-3-3 isoforms and HSPB6 dimers, but only the complex formed with 14-3-3 sigma yielded crystals that could be utilized for the purpose of their research.

\subsection{Reproductive Tissues}

Binding partners of the 14-3-3 proteins, as studied in female mice, include CDC25B phosphatase in mammalian oocytes and Follicle Stimulating Hormone (FSH) receptors in granulosa cells. It is known that in amphibians and mammals, CDC25B phosphatase is phosphorylated by Protein Kinase A (PKA; kept active in oocyte cytoplasm by high levels of cAMP), and thereafter is bound to and sequestered by 14-3-3 in the oocyte cytoplasm to maintain the oocyte arrested at prophase I of meiosis [38]. CDC25B, phosphorylated at Ser321 by PKA, is bound by 14-3-3 epsilon, to regulate blockage of mouse oocytes at meiosis prophase I and fertilized mouse eggs at mitosis [39, 40]. All seven isoforms exist in mouse oocytes and eggs and bind with CDC25B phosphatase to varying levels; however, interactions of CDC25B with 143-3 epsilon and eta are not required for normal oogenesis or oocyte maturation or early embryogenesis [23]. In granulosa cells, it is 14-3-3 tau that binds to the FSH receptors and plays a role in cell signaling and survival [41]. In spermatocytes of the male reproductive system, also studied in mice, 14-3-3 theta and zeta interact with Ccna1 to inhibit apoptosis [28]. 


\subsection{Brain}

The 14-3-3 protein's interaction with human Tau protein in the brain is associated with the development of neurodegenerative disorders such as Alzheimer's and Parkinson's diseases. Protein kinase A phosphorylates key binding locations on the Tau protein, preparing it for interaction and tight binding with 14-3-3 [42].

\subsection{Cardiovascular System}

The 14-3-3 protein interacts with multiple phosphoserine-dependent binding sites within glycoprotein IbIX complex (GPIb-IX), a major receptor for platelet adhesion [43]. In recombinant systems, activity of Kv11.1 potassium ion channel is modulated by association of $\beta_{1}$-adrenergic receptors to 14-3-3 [44].

\subsection{Skin}

In research conducted on mice, it was evident that 14-3-3 sigma interacts with the keratin 14 (K14) protein in the epidermis of the skin; K14 is an intermediate filament protein that provides structure to basal keratinocytes, and interaction with 14-3-3 sigma allows it to participate in regulation of epidermal homeostasis and strengthen the skin as a protective barrier [29].

\section{Binding Partners of 14-3-3 in Various Developmental Stages}

The seven 14-3-3 isoforms that are found in mammals have variable levels of expression, functions, and protein interactions in multiple developmental stages. This study covers mitosis, meiosis, and apoptosis as they pertain to developmental stages of the cell cycle. Other developmental topics in mammals being explored include neurogenesis and neural migration, oocyte maturation, and spermatogenesis.

\subsection{Mitosis}

The 14-3-3 proteins interact with diverse binding partners across the cell cycle. Progression from G1 to S phase during mitosis can be inhibited by binding of 14-3-3 epsilon to CDC25A which holds the latter protein in the cytoplasm and holding the oocyte arrested at prophase I, preventing premature cell cycle progression. Binding of 14-3-3 sigma to the cyclin dependent kinases CDK2 and CDK4 also prevents entry into the S phase. Conversely, 14-3-3 proteins can also bind to the CDK inhibitor p27 which causes cell cycle progression [2]. The progression from G2 to $\mathrm{M}$ phase is paused by the interaction between 14-3-3 and CDC25C, also by keeping it in the cytoplasm, preventing CDC2 dephosphorylation, and creating a G2/M checkpoint [2]. Following that checkpoint, CDC25C can dephosphorylate and activate CDC2 which initiates entry into $\mathrm{M}$ phase.

\subsection{Apoptosis}

The 14-3-3 proteins undergo phosphorylation-dependent binding with the proteins BCL2-associated agonist of cell death (BAD), FOXO, and Death Associate Protein Kinase 2 (DAPK2), as well as interaction with the Raf-1 proto-oncogene, which occurs to inhibit the pro-apoptotic effects of those proteins, and subsequently, inducing cell survival [28]. As previously mentioned above, release of the apoptosis inducing protein BAX in neuroblastoma cells occurs due to the interaction between 14-3-3 and BAP1 [34]. Another study by mentions the ability of 14-3-3 to bind directly to non-phosphorylated BAX proteins [13].

\subsection{Neurogenesis and Neural Migration}

Neurogenesis is the production of neurons from neural stem cells, and neural migration is the movement of these new neurons to their functional location. Binding partners of different 14-3-3 isoforms and how they are involved with the development of the nervous system, have been revealed [13]. The isoforms 143-3 epsilon and zeta were found to participate in phosphorylation-specific binding with $\delta$-catenin, which prevents its degradation so the $\delta$-catenin protein can associate with other downstream elements of neurogenesis and the neuronal differentiation pathway. The 14-3-3 epsilon isoform also binds to CDK $5 / \mathrm{p} 53$ phosphorylated Ndel1, which regulates neural migration by impacting the downstream binding 
Taylor R. Covington and Santanu De, Adv. J. Grad. Res.; Vol. 10, Issue 1, pp: 16-22, July 2021

of Ndel1 to LIS1. The 14-3-3 gamma isoform interacts with cytoplasmic linker proteins (CLASPs) that regulate microtubule density, length, and stability. This interaction can alter the properties of microtubules during neural migration.

\subsection{Meiosis}

There is evidence that 14-3-3 interactions also impact the progression of meiosis [45]. According to several published projects detailed above, 14-3-3 binds to cytoplasmic, phosphorylated CDC25B phosphatase and prevents germinal vesicle breakdown (GVBD), maintaining oocytes arrested at meiosis prophase I. Besides, 14-3-3 eta was observed to colocalize and interact with $\alpha$-tubulin at the meiotic spindle of metaphase IIarrested mouse eggs [27]. Together, these binding partners could potentially aid in the regulation of microtubule assembly.

\subsubsection{Oogenesis and Oocyte Maturation}

As previously detailed, 14-3-3 eta appears to colocalize and interact with $\alpha$-tubulin at the metaphase II spindle region of mouse eggs in order for normal meiotic spindle formation to occur during oogenesis [27]. Other research on oocyte maturation in mice by identified that all seven isoforms are present in oocytes and eggs, and that they all interact with CDC25B in oocytes as well as eggs; however, 14-3-3 epsilon and eta are not needed for normal mouse oogenesis, oocyte maturation, fertilization, or early embryogenesis [23].

\subsubsection{Spermatogenesis}

A project executed on spermatogenesis in mice [28] found that 14-3-3 zeta and theta are Ccna1-interactors. Spermatocytes that lack enough Ccna1 undergo late meiotic prophase arrest, resulting in apoptosis. The 14-3-3 interaction acts by inhibiting apoptosis and is crucial for meiotic progression and spermatogenesis. On the other hand, protein phosphatase $1 \gamma 2$ (PP1 $\gamma 2$ ), phosphoglycerate kinase-2 (PGK2), spermatogenesis associated 18 (SPATA18), testis specific gene A-2 (TSGA-2), piwi homolog 1, dead box polypeptide 4 (DDX4), protein kinase NYD-SP25, and EAN57 have been noted to be prominent interactors of 14-3-3 in mouse testis [46]. Proteins important in metabolism and signaling have been found by proteomic analysis of 14-3-3-binding partners in bovine sperm [47]. Protein 14-3-3 zeta binds to the protein phosphatase, PP1g2 in bovine epididymal spermatozoa [48]. Recently, it was suggested that 14-3-3 epsilon alone may be required for normal sperm function [49, 50].

\section{Conclusions}

The 14-3-3 proteins are a highly conserved and ubiquitous family of eukaryotic proteins with a vast number of binding partners and regulatory roles. Not many published reviews have encompassed research specifically demonstrating the expression, distribution, or biological value of individual binding partners of these central adapter proteins, 14-3-3. The present article, for the first time, provides a comprehensive literature survey of functionally significant examples of 14-3-3 interactors in mammals, discovered by previous laboratory research. Identification of binding partners of 14-3-3 and their interactions is important to unravel the complex molecular mechanisms underlying their control of critical cellular processes, as well as for their potential application in the diagnosis, prognosis, and/or treatment of diseases. This study focuses solely on mammalian 14-3-3-binders, though several non-mammalian organisms also are known to contain these proteins. Moreover, this review highlights the established findings on 14-3-3-interactors in mammals, though many other 14-3-3-binding proteins have been detected but are yet to be investigated in detail. Recommended future research would focus on elucidating the ability and affinity of the 14-3-3 isoforms to bind with all identified target proteins in mammalian as well as non-mammalian species, cells, tissues, and developmental stages. This would enable a better understanding of isoform-specific interactions of 14-3-3s with their binding partners. As further research is undertaken on 14-3-3s and additional binding partners are discovered, this paper forms a foundation for elucidating isoform-specific interactions of these proteins. 


\section{Competing Interests}

The authors declare that there are no competing interests involved.

\section{How to Cite this Article:}

T. R. Covington and S. De, "Binding Partners of 14-3-3 (YWHA) Protein Isoforms among Mammalian Species, Tissues, and Developmental Stages", Adv. J. Grad. Res., vol. 10, no. 1, pp. 16-22, Mar. 2021.

\section{References}

[1] M. Uhart and D. M. Bustos, "Human 14-3-3 paralogs differences uncovered by cross-talk of phosphorylation and lysine acetylation," PLoS One, vol. 8, no. 2, p. e55703, 2013. 10.1371/journal.pone.0055703

[2] D. Xue, Y. Xue, Z. Niu, X. Guo, and C. Xu, "Expression analysis on 14-3-3 proteins in regenerative liver following partial hepatectomy," Genet Mol Biol, vol. 40, no. 4, pp. 855-859, Oct-Dec 2017. 10.1590/1678-4685-GMB-2017-0029

[3] X. Yang et al., "Structural basis for protein-protein interactions in the 14-3-3 protein family," Proc Natl Acad Sci U S A, vol. 103, no. 46, pp. 17237-42, Nov 14 2006. 10.1073/pnas.0605779103

[4] S. De, "The 14-3-3 (YWHA) Proteins in Signalling and Development of the Fruit Fly, Drosophila melanogaster," International Annals of Science, vol. 9, no. 1, pp. 80-85, 2020. https://doi.org/10.21467/ias.9.1.80-85

[5] N. Kumrah and S. De, "Expression and Localization of the 14-3-3 (YWHA) Protein Family within Mammals," Mako: NSU Undergraduate Student Journal, vol. 2020, no. 2, 2020, Art. no. 2. https://nsuworks.nova.edu/mako/vol2020/iss2/2

[6] N. Kumrah and S. De, "Expression \& Localization of the 14-3-3 Protein Family Within Mammals," Presentation 2020. Trick to the Treat of Internships and Research, Nova Southeastern University https://nsuworks.nova.edu/trick/2020/events/11/

[7] R. A. Mariani, S. Paranjpe, R. Dobrowolski, and G. F. Weber, "14-3-3 targets keratin intermediate filaments to mechanically sensitive cell-cell contacts," Mol Biol Cell, vol. 31, no. 9, pp. 930-943, Apr 15 2020. 10.1091/mbc.E18-06-0373

[8] Y. Xiao et al., "Characterization of the Interactome of the Porcine Reproductive and Respiratory Syndrome Virus Nonstructural Protein 2 Reveals the Hyper Variable Region as a Binding Platform for Association with 14-3-3 Proteins," J Proteome Res, vol. 15, no. 5, pp. 1388-401, May 6 2016. 10.1021/acs.jproteome.5b00396

[9] P. H. Hsu et al., "14-3-3theta is a binding partner of rat Eag1 potassium channels," PLoS One, vol. 7, no. 7, p. e41203, 2012. https://doi.org/10.1371/journal.pone.0041203

[10] E. Barley and S. De, "Functions of 14-3-3 proteins and their isoforms across various mammaliam species, cells, tissues, organs and developmental stages," Presentation 2020. Trick to the Treat of Internships and Research, Nova Southeastern University https://nsuworks.nova.edu/trick/2020/events/12/

[11] C. A. Toleman et al., "Structural basis of O-GlcNAc recognition by mammalian 14-3-3 proteins," Proc Natl Acad Sci U S A, vol. 115, no. 23, pp. 5956-5961, Jun 5 2018. https://doi.org/10.1073/pnas.1722437115

[12] M. Pozuelo Rubio et al., "14-3-3-affinity purification of over 200 human phosphoproteins reveals new links to regulation of cellular metabolism, proliferation and trafficking," Biochem J, vol. 379, no. Pt 2, pp. 395-408, Apr 15 2004. 10.1042/BJ20031797

[13] B. Cornell and K. Toyo-Oka, "14-3-3 Proteins in Brain Development: Neurogenesis, Neuronal Migration and Neuromorphogenesis," Front Mol Neurosci, vol. 10, p. 318, 2017. 10.3389/fnmol.2017.00318

[14] S. De, "Protein 14-3-3 (YWHA) isoforms and their roles in regulating mouse oocyte maturation," Kent State University, 2014. https://nsuworks.nova.edu/cnso_bio_facbooks/22/

[15] S. De and D. Kline, "The importance of 14-3-3 (YWHA) proteins in mammalian reproduction and fertility," Conference Presentation, 29th Annual Graduate Research Symposium, Kent State University 2014. https://nsuworks.nova.edu/cnso_bio_facpres/333/

[16] S. De and D. Kline, "Interactions of 14-3-3 proteins with CDC25B phosphatase in ovaries and oocytes of adult mice," Conference Presentation, 26th Annual Graduate Research Symposium, Kent State University 2011. https://nsuworks.nova.edu/cnso_bio_facpres/326/

[17] S. De, J. Marcinkiewicz, and D. Kline, "Expression of 14-3-3 Protein Isoforms in Different Stages of Follicular Development in Adult Mouse Ovaries," Biology of Reproduction, vol. 85, no. Suppl_1, p. 639, 2011. https://doi.org/10.1093/biolreprod/85.s1.639

[18] S. De, J. L. Marcinkiewicz, S. Vijayaraghavan, and D. Kline, "Expression of 14-3-3 protein isoforms in mouse oocytes, eggs and ovarian follicular development," BMC Res Notes, vol. 5, p. 57, Jan 23 2012. 10.1186/1756-0500-5-57

[19] S. De, A. Reese, and D. Kline, "Interactions of 14-3-3 (YWHA) protein isoforms with CDC25B phosphatase in mouse oocytes," Mol Biol Cell, \#1571 vol. 23, 2012. https://nsuworks.nova.edu/cnso_bio_facpres/330/

[20] S. De, A. Reese, and D. Kline, "Interactions of 14-3-3 protein isoforms with CDC25B phosphatase in mouse oocyte maturation," Conference Presentation, Duolink User Meeting 2011. https://nsuworks.nova.edu/cnso_bio_facpres/328/

[21] S. De, A. Reese, and D. Kline, "Duolink in situ proximity ligation assays reveal interactions of 14-3-3 protein isoforms with CDC25B phosphatase in mouse oocyte maturation," Conference Presentation, 27th Annual Graduate Research Symposium, Kent State University 2012. https://nsuworks.nova.edu/cnso_bio_facpres/332/

[22] S. De, B. F. Villarreal, S. Vijayaraghavan, and D. Kline, "Identification of 14-3-3 Protein Isoforms and their Differential Subcellular Distribution in Mouse Oocytes and Eggs," Mol Biol Cell \#381 vol. 22, 2011. https://doi.org/10.1091/mbc.e11-08-0667

[23] A. A. Eisa et al., "YWHA (14-3-3) protein isoforms and their interactions with CDC25B phosphatase in mouse oogenesis and oocyte maturation," BMC Developmental Biology, vol. 19, no. 1, p. 20, 2019/10/22 2019. https://doi.org/10.1186/s12861-019-0200-1

[24] A. C. Detwiler, S. De, and D. Kline, "Interactions of YWHA (14-3-3) protein isoforms with CDC25B phosphatase in regulating mouse oocyte maturation," Conference Presentation, 48th Annual Meeting of the Society for the Study of Reproduction 2015. https://nsuworks.nova.edu/cnso_bio_facpres/331/

[25] S. De, S. Davis, D. Letwin, C. Mozena, and D. Kline, "Protein 14-3-3 eta (YWHAH) is essential for normal meiotic spindle assembly during in vitro maturation of mouse oocytes," Mol Biol Cell, \#1967 vol. 23, 2012. https://nsuworks.nova.edu/cnso_bio_facpres/329/ 
Taylor R. Covington and Santanu De, Adv. J. Grad. Res.; Vol. 10, Issue 1, pp: 16-22, July 2021

[26] S. De and D. Kline, "Erratum to: evidence for the requirement of 14-3-3eta (YWHAH) in meiotic spindle assembly during mouse oocyte maturation," BMC developmental biology, vol. 14, no. 1, p. 20, 2014. https://doi.org/10.1186/1471-213X-14-20

[27] S. De and D. Kline, "Evidence for the requirement of 14-3-3eta (YWHAH) in meiotic spindle assembly during mouse oocyte maturation," BMC Developmental Biology, vol. 13, no. 1, p. 10, 2013/04/01 2013. 10.1186/1471-213X-13-10

[28] S. K. Panigrahi, M. Manterola, and D. J. Wolgemuth, "Meiotic failure in cyclin A1-deficient mouse spermatocytes triggers apoptosis through intrinsic and extrinsic signaling pathways and 14-3-3 proteins," PLoS One, vol. 12, no. 3, p. e0173926, 2017. 10.1371/journal.pone.0173926

[29] Y. Guo et al., "Keratin 14-dependent disulfides regulate epidermal homeostasis and barrier function via 14-3-3sigma and YAP1," Elife, vol. 9, May 5 2020. https://doi.org/10.7554/eLife.53165.sa2

[30] A. J. Snow, P. Puri, A. Acker-Palmer, T. Bouwmeester, S. Vijayaraghavan, and D. Kline, "Phosphorylation-dependent interaction of tyrosine 3-monooxygenase/tryptophan 5-monooxygenase activation protein (YWHA) with PADI6 following oocyte maturation in mice," Biol Reprod, vol. 79, no. 2, pp. 337-47, Aug 2008. 10.1095/biolreprod.108.069328

[31] D. Smani, S. Sarkar, J. Raymick, J. Kanungo, M. G. Paule, and Q. Gu, "Downregulation of 14-3-3 Proteins in a Kainic Acid-Induced Neurotoxicity Model," Mol Neurobiol, vol. 55, no. 1, pp. 122-129, Jan 2018. 10.1007/s12035-017-0724-y

[32] S. Ghorbani, A. Fossbakk, A. Jorge-Finnigan, M. I. Flydal, J. Haavik, and R. Kleppe, "Regulation of tyrosine hydroxylase is preserved across different homo- and heterodimeric 14-3-3 proteins," Amino Acids, vol. 48, no. 5, pp. 1221-9, May 2016. 10.1007/s00726-0152157-0

[33] C. Yu et al., "14-3-3 $\gamma$ affects eIF5 to regulate $\beta$-casein synthesis in bovine mammary epithelial cells," Canadian Journal of Animal Science, vol. 96, no. 4, pp. 478-487, 2016. https://doi.org/10.1139/cjas-2016-0038

[34] W. Sime et al., "BAP1 induces cell death via interaction with 14-3-3 in neuroblastoma," Cell Death \& Disease, vol. 9, no. 5, pp. 116, 2018. https://doi.org/10.1038/s41419-018-0500-6

[35] R. Yuan et al., "Chk1 and 14-3-3 proteins inhibit atypical E2Fs to prevent a permanent cell cycle arrest," The EMBO journal, vol. 37, no. 5, p. e97877, 2018. 10.15252/embj.201797877

[36] Z.-M. Zhong et al., "Adaptor protein LNK promotes anaplastic thyroid carcinoma cell growth via 14-3-3 $\varepsilon / \gamma$ binding," Cancer Cell International, vol. 20, no. 1, pp. 1-12, 2020. https://doi.org/10.1186/s12935-019-1090-9

[37] N. N. Sluchanko et al., "Structural basis for the interaction of a human small heat shock protein with the 14-3-3 universal signaling regulator," Structure, vol. 25, no. 2, pp. 305-316, 2017. 10.1016/j.str.2016.12.005

[38] G. Pirino, M. P. Wescott, and P. J. Donovan, "Protein kinase A regulates resumption of meiosis by phosphorylation of Cdc25B in mammalian oocytes," Cell Cycle, vol. 8, no. 4, pp. 665-70, Feb 15 2009. 10.4161/cc.8.4.7846

[39] J. Meng et al., "The Role of 14-3-3e Interaction with Phosphorylated Cdc25B at Its Ser321 in the Release of the Mouse Oocyte from Prophase I Arrest," PLoS One, vol. 8, no. 1, p. e53633, 2013. https://doi.org/10.1371/journal.pone.0053633

[40] C. Cui et al., "14-3-3 epsilon prevents G2/M transition of fertilized mouse eggs by binding with CDC25B," BMC Dev Biol, vol. 14, p. 33, Jul 25 2014. 10.1186/s12861-014-0033-x

[41] J. A. Dias, S. D. Mahale, C. A. Nechamen, O. Davydenko, R. M. Thomas, and A. Ulloa-Aguirre, "Emerging roles for the FSH receptor adapter protein APPL1 and overlap of a putative 14-3-3tau interaction domain with a canonical G-protein interaction site," Mol Cell Endocrinol, vol. 329, no. 1-2, pp. 17-25, Nov 25 2010. 10.1016/j.mce.2010.05.009

[42] K. V. Tugaeva, P. O. Tsvetkov, and N. N. Sluchanko, "Bacterial co-expression of human Tau protein with protein kinase A and 143-3 for studies of 14-3-3/phospho-Tau interaction," PLoS One, vol. 12, no. 6, p. e0178933, 2017. 10.1371/journal.pone.0178933

[43] Y. Chen, Z. M. Ruggeri, and X. Du, "14-3-3 proteins in platelet biology and glycoprotein Ib-IX signaling," Blood, vol. 131, no. 22, pp. 2436-2448, May 31 2018. 10.1182/blood-2017-09-742650

[44] A. S. Tutor et al., "Association of 14-3-3 proteins to $\beta 1$-adrenergic receptors modulates Kv11. $1 \mathrm{~K}+$ channel activity in recombinant systems," Molecular biology of the cell, vol. 17, no. 11, pp. 4666-4674, 2006. https://doi.org/10.1091/mbc.e06-05-0422

[45] S. De, "The 14-3-3 (YWHA) Proteins in Mammalian Reproduction," International Annals of Science, vol. 10, no. 1, pp. 52-59, 2020. https://doi.org/10.21467/ias.10.1.52-59

[46] P. Puri, A. Acker-Palmer, R. Stahler, Y. Chen, D. Kline, and S. Vijayaraghavan, "Identification of testis 14-3-3 binding proteins by tandem affinity purification," Spermatogenesis, vol. 1, no. 4, pp. 354-365, Oct 2011. 10.4161/spmg.1.4.18902

[47] P. Puri, K. Myers, D. Kline, and S. Vijayaraghavan, "Proteomic analysis of bovine sperm YWHA binding partners identify proteins involved in signaling and metabolism," Biol Reprod, vol. 79, no. 6, pp. 1183-91, Dec 2008. 10.1095/biolreprod.108.068734

[48] Z. Huang, K. Myers, B. Khatra, and S. Vijayaraghavan, "Protein 14-3-3zeta binds to protein phosphatase PP1gamma2 in bovine epididymal spermatozoa," Biol Reprod, vol. 71, no. 1, pp. 177-84, Jul 2004. 10.1095/biolreprod.104.027284

[49] A. A. Eisa, "Role of 14-3-3 eta and epsilon in gametogenesis," Kent State University, 2019. http://rave.ohiolink.edu/etdc/view?acc_num=kent1574096506246273

[50] A. Eisa et al., "The protein YWHAE (14-3-3 epsilon) in spermatozoa is essential for male fertility," Andrology, Jul 132020. https://doi.org/10.1111/andr.12865

Publish your books with AIJR publisher-

$\checkmark$ Publish with ISBN and DOI.

$\checkmark$ Publish Thesis/Dissertation as Monograph.

$\checkmark \quad$ Publish Book Monograph.

$\checkmark$ Publish Edited Volume/ Book.

$\checkmark$ Publish Conference Proceedings

$\checkmark \quad$ Retain full copyright of your books.

Submit your manuscript at books. aijr.org
Publish your research article in AIJR journals-

$\checkmark$ Online Submission and Tracking

$\checkmark$ Peer-Reviewed

$\checkmark$ Rapid decision

$\checkmark \quad$ Immediate Publication after acceptance

$\checkmark \quad$ Articles freely available online

$\checkmark$ Retain full copyright of your article.

Submit your article at journals.aijr.org 